\title{
Don, patriotisme et sociétés populaires en l'an II. « Le sans-culotte de l'Hérault »
}

Nathalie Alzas

\section{(2) OpenEdition \\ 12 Journals}

Édition électronique

URL : https://journals.openedition.org/ahrf/676

DOI : $10.4000 /$ ahrf.676

ISSN : 1952-403X

Éditeur :

Armand Colin, Société des études robespierristes

Édition imprimée

Date de publication : 1 septembre 2002

Pagination : 41-65

ISSN : 0003-4436

\section{Référence électronique}

Nathalie Alzas, «Don, patriotisme et sociétés populaires en l'an II. « Le sans-culotte de l'Hérault » », Annales historiques de la Révolution française [En ligne], 329 | juillet-septembre 2002, mis en ligne le 27 mars 2008, consulté le 24 avril 2022. URL : http://journals.openedition.org/ahrf/676 ; DOI : https:// doi.org/10.4000/ahrf.676 


\title{
DON, PATRIOTISME ET SOCIÉTÉS POPULAIRES EN L'AN II «LE SANS-CULOTTE DE L'HÉRAULT »
}

\author{
NATHALIE ALZAS
}

La diffusion du don patriotique est caractéristique de l'an II. Son étude, centrée sur les listes de donateurs, permet de cerner la pratique de la citoyenneté en Révolution. La diversité du don, sur le terrain, indique l'aspect volontaire de ce geste en général, en mettant en valeur les zones patriotiques. II dévoile, à la fois, le succès des sociétés politiques pour diffuser les principes révolutionnaires, et les limites de leur action. La société populaire assume, dans sa pratique de l'offrande, le rôle de la communauté d'habitants : en l'an II, la réussite des dons ne peut s'expliquer que par cet aspect, avec des sociétés politiques qui parviennent à transcender la fierté et les solidarités locales en appartenance nationale.

Mots clés : Révolution ; an II ; Hérault ; patriotisme ; don ; sociétés populaires.

Au cœur du patriotisme en Révolution, la pratique du don occupe une place essentielle, notamment en l'an II. Les innombrables dons qui parviennent à la Convention soulignent l'ampleur d'un phénomène révélateur de la mobilisation idéologique et matérielle. Des travaux pionniers ont abordé la question en s'appuyant sur les Archives parlementaires (1), pour esquisser une géographie des dons, afin de percevoir leurs proportions, leurs motivations. Tous s'accordent sur la difficulté d'une telle entreprise à l'échelle nationale, car aucune source ne comptabilise l'ensemble des dons. La seule certitude en ce domaine est la profusion de gestes qui remplissent bien des pages d'adresses envoyées à la Convention nationale. Il semble que les offrandes se

(1)Catherine DUPRAT, «Don et citoyenneté en l'an II. Les vertus du peuple français», dans Révolution et République. L'exception française. Colloque de Paris I, 21-26 septembre 1992, Paris, Kimé, 1994, pp. 263-281 ; Michel VOVELE, La découverte de la politique. Géopolitique de la Révolution française, Paris, La Découverte, 1992, pp. 254-256.

Annales historiques de la Révolution française - $2002-N^{\circ} 3$ [41 à 65] 
répartissent assez irrégulièrement sur le territoire. Elles dominent surtout dans les départements qui connaissent une forte densité de sociétés populaires. Les motivations des donateurs restent difficiles à cerner, de l'aveu même de ceux qui se sont penchés sur la question. Il est vrai qu'une enquête à partir des documents de la Convention nationale ne permet pas de savoir qui donne et comment. Les archives laissées par les municipalités et par les sociétés populaires offrent, en revanche, des listes de donateurs. Comparer ces listes avec celles des membres des sociétés politiques et des administrations municipales, leur correspondance, permettrait de percevoir l'impact du don parmi les populations. L'omniprésence de ce geste patriotique pose des questions sur son enracinement. Pour saisir ses modalités, le profit du donateur ne suffit pas. Le citoyen est inséré dans une communauté qui doit être animée d'une dynamique particulière pour que l'offrande ne soit pas un acte isolé. Le poids des héritages est donc à considérer.

Pour aborder ces questions, il est nécessaire de concentrer l'étude sur un don d'envergure pour disposer de sources suffisantes, dans un espace géographique qui, quoique réduit pour la commodité de l'analyse, soit significatif. Cette étude est centrée sur le département de l'Hérault. Situé sur la Méditerranée, issu de l'ancienne province de Languedoc, dans un Midi plutôt agité, ce département apparait patriote, avec un réseau dense de sociétés politiques (2). Il constitue un terrain d'étude intéressant pour suivre le don qui a connu la plus grande extension en l'an II, l'offrande d'un vaisseau de ligne à la patrie.

\section{Le don d'un vaisseau et ses antécédents}

Les souscriptions pour la construction d'un navire voient le jour pendant l'hiver de l'an II. Les sociétés populaires affirment avec éclat la vigueur de leur engagement politique. Elles font des souscriptions au niveau départemental en mettant en commun l'argent recueilli. En quelques jours, elles réussissent à récolter des sommes considérables (3). Chaque département souhaite recueillir suffisamment de fonds pour armer un vaisseau qui porte son nom. Ce n'est pas cependant une utopie révolutionnaire, car il existe un précédent que les patriotes locaux doivent garder en mémoire.

À la suite de la guerre de Sept Ans, le duc de Choiseul souhaitait reconstituer une marine française capable de prendre une revanche sur l'Angleterre. Comme toujours, l'argent fait cruellement défaut au ministère

(2) Jean-François DUBOST, «Le réseau des sociétés politiques dans le département de l'Hérault », $A H R F, 1989$, pp. 374-416.

(3) « Â Bayonne 1200000 livres, à Orléans 2000000 », d'après Louis DE CARDENAL, La province pendant la Révolution. Histoire des Clubs jacobins (1789-1795), Paris, Payot, 1929, pp. 353-354. 
de la Marine. Pour surmonter l'obstacle financier, Choiseul a recours à des souscriptions publiques qui donnent dix-huit navires au pays dans les années 1760 (4). Il en appelle à la générosité des particuliers et surtout à celle des corps constitués. L'opération commence par une intervention directe auprès du cardinal de la Roche-Aimon, archevêque de Narbonne, et donc président des États de Languedoc (5). Choiseul est écouté : l'archevêque propose, dans la séance du 26 novembre 1761 des États de Languedoc, «d'offrir à sa majesté un vaisseau de ligne de 74 pièces de canon et de donner par cette démarche au reste de la France (...) le signal de ce que peuvent et doivent faire les sujets véritablement dignes du meilleur des maîtres (...) » (6). Les États de Languedoc se donnent en exemple. Ils sont suivis par ceux d'Artois, de Provence, de Flandre, de Bourgogne, des villes de Paris, Bordeaux, des marchands des six corps de Paris, de la chambre de commerce de Marseille, des fermiers généraux, etc. (7). Chaque vaisseau prend le nom de son donateur. L'archevêque de Narbonne attribue celui de Languedoc au navire payé par les États. Ces derniers contractent des emprunts pour réunir des fonds, le premier se portant à 400000 livres (8). Même si certaines souscriptions lancées pour l'offrande des vaisseaux sont peut-être des emprunts masqués, il est indéniable qu'elles représentent un succès pour la marine et pour Choiseul (9). Elles donnent lieu à un élan patriotique qu'il ne faut pas surestimer mais qui est très réel. Des expressions utilisées par le cardinal de la Roche-Aimon lors de son discours de 1761 peuvent être rapprochées d'autres développées par les sociétés populaires bien plus tard : « Il n'est point de bon Français qui ne se sente animé du désir de tout sacrifier pour concourir » aux efforts du roi et du «ministre sage et éclairé » pour restaurer la marine française (10). L'exaltation du sacrifice dans le don, au nom d'un intérêt supérieur, est déjà présent. La grande différence entre les discours de l'un et des autres est le remplacement de terme «roi» par «patrie ». Bien plus, des gestes civiques des citoyens de l'an II ne sont pas sans rappeler, par leur forme et la façon dont ils sont accueillis, ceux des sujets de 1761 :

«Non seulement les provinces donnèrent, dans cette occasion, des marques distinguées d'un zèle rare, mais M. de Choiseul m'a dit qu'il recevait

(4) Histoire militaire de la France (direction André Corvisier), t. 2, Paris, PUF, 1992, p. 177.

(5) Mémoires du Baron de Besenval sur la cour de France, Paris, 1987 pour la dernière édition, Mercure de France. «Don que les provinces firent au roi de vaisseaux et d'argent », pp. 122-124 (écrit en 1772).

(6) Discours de l'archevêque dudit jour, ADH (Archives départementales de l'Hérault), C 7530, folios 160 et 161 .

(7) Martine ACERRA et Jean MEYER, Histoire de la marine française, Paris, 1994, Éditions OuestFrance, p. 113.

(8) ADH, C 7530. Discours de l'archevêque devant les États, 26 novembre 1761.

(9) Histoire militaire de la France, op. cit., tome II, p. 177.

(10) ADH, C 7530. Discours de l'archevêque devant les États, 26 novembre 1761. 
journellement des lettres de particuliers qui lui offraient de l'argent. Il en eut une entre autres d'un simple gentilhomme de Champagne du nom duquel malheureusement il ne s'est pas souvenu, et qui lui mandait que n'étant pas riche et ayant des enfants, il n'était pas trop en état de donner; que cependant, comme ils étaient en bas âge, il pouvait se passer de mille écus qu'il avait amassés et qu'il les lui envoyait pour être employés au service du roi. M. de Choiseul lui répondit que sa Majesté, après les avoir acceptés, les lui restituait pour qu'ils aidassent à l'éducation de ses enfants, qui ne pouvaient manquer, avec un tel père, de lui rendre de grands services (...) » (11)

La pratique du don connaît pendant la Révolution une extraordinaire floraison qui a été préparée par la diffusion de certaines valeurs pendant tout le XVIII ${ }^{\mathrm{e}}$ siècle qui aboutissent à l'exaltation du sacrifice librement consenti pour la patrie. Témoin l'attitude des membres des États de Languedoc en 1761 :

« (...) Messieurs les évêques et barons ici présents ayant déclaré d'avance qu'ils entendent céder leurs pensions pour servir au payement de partie des dits intérêts [de l'emprunt pour le payement du vaisseau], ce qu'ont offert pareillement les sieurs députés du Tiers État et les officiers de la province par rapport à leurs montres pour le soulagement des redevables » (12)

Les membres des États donnent l'exemple en s'impliquant dans l'offrande, comme les administrateurs locaux près de 30 ans plus tard. Les départements, en proposant par la voie des sociétés politiques, d'offrir un vaisseau à la patrie, s'inscrivent dans le sillage d'institutions dont ils ont hérité les prérogatives, du moins en partie. Chaque société populaire, comme les États de Languedoc, agit en tant que membre d'une entité souveraine, le peuple en l'an II, un corps privilégié en 1761. Le précédent de 1761, sans nul doute, joue un rôle dans l'apparition, en l'an II, sur ces mêmes terres du Languedoc, de souscriptions pour un vaisseau départemental : don de même nature, pour la défense de valeurs similaires, objectifs semblables, à partir d'un niveau local, tout en exaltant son civisme et sa petite «patrie », province ou département. Il est vrai que les difficultés de l'État sont du même ordre (le manque d'argent), ce qui incite les dirigeants à en appeler à la générosité du peuple.

Un don de si vaste envergure suscite également des interrogations sur la charge émotionnelle ou symbolique du «vaisseau» à cette époque. La Révolution réutilise une image ancienne de l'iconologie de l'État pendant les Temps modernes. Traditionnellement, la conduite du pays est souvent comparée à la direction d'un navire (13). Les rois de l'Ancien Régime ont souvent utilisé la métaphore à leur profit, en se faisant représenter avec un

(11) Mémoires du Baron de Besenval sur la cour de France, op. cil., p. 124.

(12) ADH, C 7530. Séance des États de Languedoc du 26 novembre 1761, folio 161.

(13) Iconologie ou explication nouvelle de plusieurs images, emblèmes et autres figures..., de Cesare RIPA, moralisées par Jean BAUDOIN, Paris, 1644 ; réimp. Dijon, Faton, 1999. 
timon de navire dans les mains. Pendant la Révolution, l'emploi du mot «vaisseau » est très fréquent dans les discours patriotiques. Il est employé au sens figuré pour représenter soit la République, soit la Révolution. Il connaît un grand succès qui ne se dément pas. Il est utilisé pour indiquer combien l'aventure vécue est nouvelle, irréversible, ainsi par Jeanbon SaintAndré dans une lettre à Barère en 1793 : «Il faut dire bien ouvertement à l'Assemblée nationale : vous êtes une assemblée révolutionnaire. Nous devons conduire à bon port le vaisseau de l'État ou périr avec lui » (14). Dans maints discours, le terme revient, car il permet l'identification de la Révolution à un voyage incertain sur des mers inconnues d'un équipage, le peuple, où chacun doit œuvrer pour le salut commun, après avoir rompu les amarres, celles de l'Ancien Régime, qui maintenaient prisonnier. Le « vaisseau » permet d'insister sur la coupure unique, inouïe entre deux mondes, le passé et le présent que tout sépare désormais (15). La métaphore donne au mot une consonance particulière, qui perdure pendant toute la Révolution, et qui jaillit surtout lorsque les difficultés surgissent. Ainsi, l'agent municipal de Bédarieux, en des jours difficiles de l'an VII, reprend l'expression pour y puiser une profession de foi énergique : «Que la loi seule [soit] notre boussole et la République le vaisseau qui doit nous sauver du naufrage » (16). Le «vaisseau » dans les textes officiels est donc une allégorie de l'État ou de la République et peut s'étendre aux institutions. Cela explique des emplois innombrables (17). La charge symbolique du terme ne saurait être oubliée au moment où les patriotes décident de faire des dons pour la construction de «vaisseaux». Au sens figuré comme au sens propre, le mot prend des connotations fortement patriotiques. Le don d'un vaisseau symbolise le patriotisme tout comme le mot «vaisseau » symbolise la République. Il n'est pas entièrement hasardeux d'estimer que des interactions idéologiques, entre les deux façons d'appréhender le terme, sont importantes.

Malgré cela, et le précédent des années 1760, né en Languedoc, l'idée d'offrir un navire à la patrie connaît un cheminement assez long pendant la Révolution, même si elle est présente très tôt. En mai 1790, un député de la Constituante propose à ses collègues «de sacrifier leur traitement à la confection d'un vaisseau de guerre »(18). Cette proposition a déjà été

(14) Cité par Michel VOVEUE, «L'événement », dans Combats pour la Révolution française. Paris, La Découverte, 1993, p. 199.

(15) Ibid. Cambon : « Nous avons abordé dans l'île de la Liberté et nous avons brûlé les vaisseaux qui nous y ont conduits. »

(16) Agent municipal Gabriac, 2 pluviôse an VII, à l'occasion de la fête « de la juste punition du dernier roi des Français », cité par Roger ALLAIRE, Histoire de Bédarieux, si, 1911.

(17) Lettre de l'agent national de Neffiès à celui du district de Béziers, 10 vendémiaire an III, compte rendu décadaire, à propos de la municipalité qu'il considère comme incapable et dont il demande la destitution : "Citoyen, si tu ne confies pas le gouvernail à des mains plus habiles, le vaisseau échouera », $\mathrm{ADH}, \mathrm{L} 4048$.

(18) Courrier d'Avignon, n 136,8 juin 1790. 
soumise plusieurs fois à l'Assemblée. L'initiative, loin d'être louée, est vigoureusement rejetée. Des journaux patriotes blâment la proposition en adoptant une critique sévère : « (...) Le projet que j'ai à vous proposer, disoit ce député, aux représentants de la Nation vous couvrira de gloire. Ce qui les couvrira de gloire, c'est la Constitution qu'ils ont avancée, qu'ils vont achever \& qu'ils n'auraient pas pu même commencer si dénués de fortune, comme ils le sont pour la plupart, ils n'avaient pas été soutenus par le traitement qu'ils reçoivent » (19).

La rupture idéologique entre le début de la Révolution et l'an II est nette. Les priorités ne sont pas les mêmes, la façon de considérer les représentants du peuple non plus. Entre ces deux périodes, il y a une évolution profonde de la société française, sur le plan politique et social, qui fait du patriotisme la valeur essentielle.

\section{Naissance et organisation d'un don : le sans-culotte de l'Hérault}

Le projet d'un vaisseau départemental est un des dons patriotiques qui a laissé le plus de traces dans les archives de l'Hérault. Sa date tardive explique qu'il n'a pas été suivi d'aucun commencement d'exécution, même si de nombreuses offrandes sont recueillies dès germinal an II. Les dons, versés dans les caisses du receveur du département, se trouvent de facto entre les mains de l'État, à partir du moment où, après Thermidor, l'affaiblissement des sociétés populaires ne permet plus la réalisation de leurs ambitions. Le gouvernement thermidorien, ayant supprimé le droit des sociétés politiques à réaliser des souscriptions, entreprend un inventaire de toutes les sommes restant en caisse (20), ce qui a permis de conserver dans les archives, des listes entières de donateurs, par sociétés populaires ou par villes. Ces listes, ajoutées à la correspondance et aux séances des sociétés politiques, permettent de percevoir les réalités d'un don sur l'ensemble d'un département, loin des mentions ponctuelles souvent évoquées pour quelques sociétés populaires, ou d'une analyse nationale qui reste très générale. La souscription pour le vaisseau est en outre une des rares offrandes qui est réalisée par l'ensemble des sociétés politiques d'un département donné, pour remplir une caisse commune. Cela ouvre d'autres perspectives que les dons qui ne concernent que les sociétés populaires prises isolément, chacune entreprenant des collectes particulières, ce qui donne des bilans, qui, faute de points de comparaisons, ressemblent à des inventaires à la Prévert.

(19) Ibid.

(20) Selon le décret du 13 frimaire an III. 
Dans l'Hérault, l'initiative revient à la société populaire de Montpellier. Cette dernière décide, le 17 nivôse an II, d'ouvrir une souscription pour construire un vaisseau qui serait baptisé l'Hérault, ou mieux encore, le sansculotte de l'Hérault (21). Déjà de nombreuses sociétés politiques ont lancé une telle souscription en recommandant à leurs homologues de les imiter. Les événements de Toulon expliquent le projet. Les pertes de la flotte française sont durement ressenties par les patriotes inquiets. Malgré la reprise du port, les craintes sont vives de voir « la perfide Albion » posséder «l'empire des mers». La société populaire de Montpellier décide de lancer sa souscription à la suite d'une adresse de son homologue d'Annonay, semblet-il (22). L'offrande d'un vaisseau départemental dans l'Hérault est une initiative conduite de bout en bout par la société populaire de Montpellier. Elle est la première à évoquer la souscription, qu'elle entend diriger. Elle envoie aux sociétés populaires du département, aux agents nationaux des districts une adresse imprimée, pour être imitée aussi bien par les sociétés politiques que par les municipalités (23). Quelques semaines plus tard, la société populaire de Montpellier expédie une nouvelle adresse dans tout l'Hérault afin de convaincre les retardataires ou les réticents. La plupart des offrandes sont envoyées à la société populaire de Montpellier, qui les reçoit de nivôse à thermidor an II (24). Les dons sont centralisés dans la caisse du receveur du département, chargé de délivrer à chaque organisation donatrice un .bordereau. Dans la correspondance de la société populaire de Montpellier qui subsiste, trente lettres de sociétés populaires ou de municipalités précisent les conditions dans lesquelles elles ont décidé de faire une souscription pour le vaisseau (25). Sur trente, vingt-quatre disent que la décision est prise immédiatement après lecture de l'adresse de leur homologue montpelliéraine (26). Elles se placent directement sous son égide (27).

(21) ADH, L 5502. Registre des séances de la société populaire de Montpellier.

(22) Ibid., L 5537. Adresse imprimée de la société populaire d'Annonay, du 10 nivôse an II.

(23) Ibid., L 5502. Séance de la société populaire de Montpellier du 23 nivôse an II.

(24) Ibid., L5502-5504. Registres des procès-verbaux de la société populaire de Montpellier, vendémiaire an H-vendémiaire an III.

(25) Ibid., L 5531-L5536. Correspondance envoyée par les sociétés populaires et municipalités du département à la société populaire de Montpellier (classement par ordre alphabétique).

(26) Exemple : « (...) Après lecture de votre lettre relative à la construction d'un vaisseau de ligne (...) ; notre société populaire, animé du vif désir de concourir à la défaite à la défaite entière des ennemis de notre liberté (...) a de suite délibéré d'ouvrir une souscription (...) », lettre de la société populaire de Cessenon à celle de Montpellier, 23 ventôse an II, Ibid., L 5532.

(27) Ibid., L 5536. Lettre du Conseil général et de la société populaire des sans-culottes de SaintThibéry à la société populaire de Montpellier, 12 germinal an II. « (...) Nous avions prévu depuis longtemps l'invitation que vous nous avés faite de contribuer à la construction d'un vaisseau, nous vous aurions même prévenus si nous eussions cru notre voix assés forte pour nous faire entendre ; nous avions compté sur votre patriotisme et nous avons attendu impatiemment le cri de ralliement qui devoit sortir de votre sein du moment que nous l'avons entendu et qu'il nous a été manifesté par votre lettre (...) nous avons ouvert une souscription à l'effet de seconder votre zèle. » 
Une prééminence est accordée à la société populaire du chef-lieu du département. Par leur don, les sociétés politiques héraultaises désirent démontrer leur patriotisme à celle qui est présentée comme un guide. Mais cela ne signifie pas que les Montpelliérains désirent contrôler les autres sociétés populaires du département. D'abord l'existence même de certaines d'entre elles leur échappe, comme aux agents nationaux d'ailleurs, qui ne savent pas toujours le nombre exact de clubs qui existent dans leur circonscription (28). La société populaire de Nisas ne reçoit pas l'adresse de Montpellier sur le vaisseau départemental par exemple. Elle n'apprend l'initiative, à laquelle elle compte participer, que par «voie indirecte» (29). Enfin des sociétés populaires entendent contrebalancer le rôle de Montpellier en jouant à leur tour un rôle de guide sur les sociétés populaires ou municipalités environnantes. Béziers, qui tente toujours de concurrencer la ville de Montpellier, sa rivale, ne manque pas d'envoyer des commissaires dans les communes voisines, même celles qui sont dotées de sociétés populaires (30). D'autres, à une échelle plus modeste, imitent les plus grandes. La collecte quitte alors le cadre de la société populaire pour se répandre dans des communes moins politisées. La société politique de Lansargues envoie ainsi des commissaires pour faire procéder à la levée de fonds dans les municipalités ou hameaux proches (31). L'offrande fait office de preuve de civisme. Elle permet de solliciter une précieuse affiliation ou nouvelle reconnaissance après des tempêtes politiques (32). La liberté du don à première vue apparait limitée. Les sociétés populaires, tout comme les municipalités, ont intérêt à montrer de la bonne volonté si elles veulent être bien considérées par une organisation, la société politique de Montpellier, qui joue un rôle fondamental auprès des représentants du peuple, notamment dans les épurations. L'insistance est mise pour recevoir une reconnaissance de son patriotisme: "Quoique la somme de la commune soit modique, elle ose espérer qu'il lui sera accordé la mention civique (...) » (33). Mais des sociétés populaires, comme des municipalités, répondent à la souscription sans être vraiment sollicitées. Si elles le font, c'est qu'elles sont à l'écoute des initiatives patriotiques et s'y montrent sensibles. Cela est logique parce qu'elles sont par nature des associations patriotiques.

(28)Jean-François DUBOST, «Le réseau des sociétés politiques dans le département de l'Hérault... », op. cit. p. 403.

(29) ADH, L 5534. Lettre du 13 floréal an II de la « société populaire des sans-culottes de Nisas » à la société populaire de Montpellier.

(30) Archives municipales de Béziers, II J. Séances de la société populaire de Béziers du printemps de l'an II. Concerne Capestang, Cajouls, Camplong, Nissan, Boussagues.

(31) Touche « Saint-Côme, Valergues, Mudaison, Saint-Nazaire », ADH, L 5533. Extrait du registre de la « société populaire des vrais montagnards de Lansargues », 12 et 25 germinal an II.

(32) Ibid., L 5531. Lettre (sans date) de la « société populaire des sans culottes de Bessan » à celle de Montpellier pour « renouveler son affiliation », et qui ne manque pas d'énumérer le don pour le vaisseau, diverses offrandes pour les soldats et un cavalier jacobin.

(33) Ibid., L 5532. Extrait des séances de la commune de Creissan du 23 floréal an II. 
L'offrande est une de leurs raisons d'être. Mais le don n'est pas l'affaire des seules sociétés populaires. Il existe une coordination étroite entre elles et les autorités du cru. La société politique de Montpellier sollicite autant les agents nationaux des districts que ses homologues pour inciter la population. Elle sait qu'elle peut compter sur eux, qui se targuent de déployer un grand zèle (34). L'offrande pour le vaisseau indique la solidité des liens qui unissent les sociétés politiques à leur municipalité. À Saint-Thibéry, le conseil général de la commune se « réunit à la société populaire des sansculottes de ladite commune afin de prendre une décision commune concernant la souscription, qui est faite dans une séance extraordinaire des deux organisations, puisqu'elles sont toutes deux réunies » (35). Le procès-verbal de la séance est signé à la fois par le maire, les officiers municipaux, et le bureau du club. L'offrande permet de discerner les relations entre sociétés populaires et municipalités. On peut se demander si de telles séances n'indiqueraient pas une quasi-fusion en certains lieux entre municipalités et sociétés politiques, qui ressemblerait à une assemblée générale des habitants. Des affirmations de sociétés populaires pourraient le laisser penser : «nous nous sommes joints au conseil général de notre commune qui ne fait qu'un avec nous » (36). Cette interrogation peut être également posée à propos d'autres décisions, celles concernant les réquisitions militaires par exemple. Mais le don, s'il permet de montrer des communautés soudées, patriotes, dévoile aussi les tensions politiques et sociales qui déchirent des communes. Il devient une arme dans les règlements de compte locaux ayant pour objectif l'affirmation du pouvoir d'une partie du village ou du bourg sur une autre, incessant combat qui traverse toute la Révolution et les siècles. Souvent, un camp se trouve à la société politique, l'autre à la municipalité. Le don permet l'expression des oppositions. Il facilite l'appel à l'extérieur pour mieux dénoncer l'adversaire. À Magalas cela est net. Le commissaire désigné pour porter l'offrande à la société populaire de Montpellier est chargé «en outre (...) de faire connaître le modérantisme et l'égoïsme de partie de notre municipalité quoique invitée à souscrire [n'a pas répondu] » (37).

(34) « (...) Frères et amis. J'ai reçu votre lettre du 4 ventôse avec plusieurs exemplaires de votre adresse aux sociétés populaires et aux communes de ce district; et je me féliciterai de pouvoir encore par ce moyen, concourir à l'exécution d'un projet aussi grand que décisif pour l'affermissement de la République et le triomphe de la liberté et de l'égalité. Salut et fraternité», ibid., L 5533. Lettre du 7 ventôse an II de l'agent national du district de Lodève à la société populaire de Montpellier. an II.

(35) Ibid., L 5536. Procès-verbal de la séance du conseil général de la commune du 12 germinal

(36) Ibid., L 5536. Lettre de la société populaire de Saint-Thibéry à celle de Montpellier, 12 germinal an II.

(37) Ibid., L 5534. Extrait des registres de la société populaire et montagnarde de Magalas, s.d., séance publique. 
Le don est geste patriotique donc politique. Il s'insère dans le jeu du pouvoir local et participe au positionnement de chacun. Il ne saurait être réduit à l'expression d'une pression extérieure, de propagande du gouvernement ou du Club des jacobins. Chaque société populaire, chaque municipalité, chaque citoyen, adopte une attitude face au don qui correspond à des opinions sociales ou politiques précises. Le don est à la fois créateur d'unité et de division. Certes, le conflit reste souvent larvé. Le refus du don patriotique ne saurait être chose facile vu la situation difficile dans laquelle se trouve le pays, et la proclamation comme valeur essentielle de l'offrande à la Patrie. Mais le silence, l'indifférence, peuvent être plus facilement assumés. Le conflit avec la société politique organisatrice du recueil des dons est alors plus feutré, mais n'en dévoile pas moins la force des antagonismes. Si la société populaire de Servian mentionne à celle de Montpellier « observation à nos frères que la susdite somme n'a été levée que dans la société et non dans la commune », la remarque n'est pas anodine (38). Des relations exécrables percent çà et là, quand ce ne sont pas des rapports inexistants (39). La pratique du don peut alors renforcer la position d'un des deux camps en présence, à savoir la société politique dans la plupart des cas.

Les hiérarchies ou antagonismes se font aussi au niveau des discours. Dans les correspondances, l'orthographe aléatoire révèle les différences qui existent entre sociétés populaires des grandes villes, fortement francisées, politisées, et les petites sociétés ou municipalités. Pour ces dernières, le français employé est parfois mêlé à des mots ou des tournures occitanes. Si l'emblématique du mot «vaisseau » peut être valable pour les sociétés politiques d'importance, ce n'est sans doute pas le cas pour des bourgades pour lesquelles le français est chose compliquée, même pour le secrétaire-greffier du lieu. Le vaisseau devient «veseau» à Candillargues (40), «vaisso » à Clapiers (41), «vaissaux» à Brissac (42), «vessaut» à Boisseron (43). Il ne faut cependant pas pousser la démonstration trop loin. S'il est évident que le français n'est pas facile à manipuler en certaines communes, cela ne signifie pas que l'implication révolutionnaire soit absente. Certes, on pourrait supposer que la construction et même l'idée d'un vaisseau est plus une cosa mentale qu'une réalité pour de nombreux villageois qui n'ont jamais vu un port ou la mer de leur vie. En ce cas, le don pourrait être plus la forme d'une allégeance

(38) Ibid., L 5536. «Extrait des délibérations de la société populaire de Servian affiliée à celles des jacobins de Paris, Montpellier, etc. extrait du 24 germinal an II ».

(39) Lettre de la société populaire des sans-culottes de Nisas à celle de Montpellier : « Nous ignorons si notre municipalité a reçu cet avis » [l'adresse invitant à la souscription ], 13 floréal an II, Ibid., L 5534.

(40) Ibid., L 3491. État des donateurs fourni par la municipalité de Candillargues, 23 ventôse an III.

(41) Ibid, pour Clapiers, 19 prairial an II.

(42) Ibid., L 5532. Lettre de la société populaire de Brissac à celle de Montpellier, 17 floréal an II.

(43) Ibid, L 3491. État fourni par Boisseron, s.d. 
à ce que l'on considère comme une autorité supérieure, la société populaire de Montpellier, mêlée peut-être dans les esprits à l'administration, que véritablement un geste patriotique assumé. Mais ce serait fortement sous-estimer la capacité de réflexion de citoyens pour qui la Révolution et la guerre font connaître leurs effets concrets, qu'ils soient bons ou mauvais. Si beaucoup de lettres se contentent de signaler à la société populaire de Montpellier le montant du don offert par une municipalité ou une société politique, d'autres développent toute une argumentation pour indiquer la nécessité de la lutte et leur motivation pour la construction du vaisseau. De nombreuses phrases stéréotypées ou empruntées à l'adresse de Montpellier reviennent, ce qui ne veut pas dire d'ailleurs qu'elles ne correspondent pas à des sentiments patriotiques profonds. La sobriété est souvent la règle : don pour le «vaisseau destiné à la défense de la patrie » (44) ou «pour la défense de notre chère liberté » (45), «pour chasser promptement nos ennemis du territoire français » (46). L'idée principale de la majorité des lettres est la volonté de libérer le territoire. L'ennemi est décrit davantage comme « un tyran » (47) que comme un étranger. Certes, la «fière Albion» (48), «les féroces anglais » (49), «brigands des mers » (50) sont dénoncés, mais l'argumentation est avant tout politique, elle n'est pas vraiment xénophobe. La lutte est entreprise au nom de la défense des territoires et des valeurs républicaines. Si l'ennemi étranger est présenté sous un jour très négatif, il n'est pas le seul dénoncé : l'interaction entre guerre et lutte contre l'ennemi intérieur est très présente (51). Le sentiment que la trahison est toujours prête, comme à Toulon, à vendre le pays est omniprésent, tout comme l'idée d'un encerclement. L'idée de construction d'un vaisseau départemental s'inscrit dans un double combat contre l'ennemi extérieur et intérieur. L'opposition n'est pas entre Français et étrangers, mais entre révolutionnaires et contre-révolutionnaires. Il est symptomatique de voir, lorsqu'il s'agit de soutenir un don pour lutter contre l'ennemi étranger par excellence, l'Anglais, que de nombreuses sociétés populaires, souvent de petite taille, mettent en avant la lutte contre des adversaires intérieurs, au nom de raisons politiques mais aussi et surtout sociales. La guerre étrangère prend l'aspect d'un prolongement de guerre civile, nourri par les tensions sociales. Les oppositions patriotes / aristocrates, révolutionnaires / tyrans, sont indissociables du conflit entre les « riches » et

(44)Ibid, L 5532. Lettre de la société populaire de Clermont-l'Hérault à celle de Montpellier, 21 pluviôse an II.

(45) Ibid, lettre de la société populaire de Cessenon, 15 germinal an II.

(46) Ibid., L 5534. Lettre de la société populaire des Matelles à celle de Montpellier, 15 floréal an II.

(47) Ibid, L 5535. Lettre de la société populaire de Gabian à celle de Montpellier, s.d.

(48) Ibid, L 5536. Lettre de la société populaire de Vernodure à celle de Montpellier, 17 germinal an II.

(49)Ibid, L 5534. Lettre de la société populaire de Marsillargues à celle de Montpellier, $1^{\mathrm{er}}$ messidor an II.

(50) Ibid, L 5536. Lettre de la société populaire de Saint-Thibéry, 12 germinal an II.

(51) Ibid, L 5535. Lettre de la société populaire de Pérols à celle de Montpellier, s.d. 
les « pauvres » (52). Par un don commun, les patriotes montrent leur cohésion face à leurs adversaires. D'ailleurs, plus qu'une désignation de l'ennemi, l'offrande s'accompagne de l'autoportrait que dressent les sociétés politiques. La présentation du don se fait l'éloge du don librement consenti.

Le geste patriotique devient célébration. Les sociétaires accueillent avec «joie», «enthousiasme», «applaudissements» l'adresse de la société populaire de Montpellier qui propose d'ouvrir une souscription pour le vaisseau (53). Le don accompagne le sacrifice que font les soldats de leur vie. La remise du don des sociétés populaires à celle de Montpellier se fait selon un cérémonial qui a pour objectif de mettre en valeur le geste patriotique et de resserrer les liens entre citoyens. Les députés d'une société politique qui remettent l'offrande dans une séance de celle de Montpellier reçoivent tous des marques d'honneur qui suivent toujours les mêmes règles : « l'accolade fraternelle », les « honneurs de la séance », avec les députés qui sont installés à la droite du président et qui, parfois, font un discours à la tribune (54). Les sociétés populaires se reconnaissent mutuellement. Cela conforte le patriotisme des petites bourgades, notamment de leurs dirigeants. La cohésion entre les patriotes en est renforcée, ainsi que la hiérarchie qui existe entre les sociétés populaires. Mais cela n'indique pas pour autant quels sont les citoyens qui donnent. Il est temps de s'intéresser à eux.

\section{Les donateurs}

Le don pour le vaisseau est un succès. La société populaire de Montpellier, dans une adresse du 4 messidor an II, annonce à la Convention une collecte qui se monte, pour le moment, dans le département, à 172259 livres (55). Le don touche au moins 116 communes sur les 334 que compte l'Hérault (56). La façon dont la société populaire de Montpellier et

(52) Ibid., L 5592. Lettre de la société populaire de Montagnac, à celle de Montpellier, 29 pluviôse an II : « (...) Ne pensez pas, frères et amis, que ce soit cette classe d'hommes riches, dont le patriotisme ne consiste qu'en un égoïsme affreux qui ayent le plus contribué, c'est au contraire ces hommes ingénieux dont la simplicité et l'amour naturel qu'ils ont pour le bien de la Patrie, les ont engagé à partager le fruit de leurs sueurs journaliers avec vous (...) ; les tigres spéculateurs de nos besoins rougeroit peut etre en réfléchissant que des hommes libres quoi que ruinés par leurs escroqueries savent par leur économie procurer à leur mère bienfaitrice les secours dont elle a besoin (...)».

(53) Parmi beaucoup d'autres : «accueillie au milieu des plus vifs transports de joie » (société populaire de Marsillargues, extrait de la séance du 12 messidor an II, ibid., L 5534); «combien nous avons ressenti de la joye » (Lettre de la société populaire de Bessan, ibid, L 5592).

(54) Ibid, L 5503-5504. Procès-verbaux des séances de la société populaire de Montpellier, pluviôse an II-vendémiaire an III.

(55) Ibid., L 5555. Avis de réception du Comité des dépêches de la Convention du 19 messidor an II de l'adresse de la société populaire de Montpellier.

(56) Ce chiffre est obtenu par recoupement des listes fournies aux districts en l'an III [ibid., L 3491, L 3495-3496, L 4180] avec la correspondance [ibid, L 5525 et L 5531-5536] ainsi que les procès-verbaux de la société populaire de Montpellier de nivôse à fructidor an II [ibid., L 5502-5504]. 
ses homologues organisent la collecte montre que les donateurs sont avant tout des membres des sociétés politiques. Il est symptomatique que la géographie des dons corresponde à celle de l'implantation des sociétés populaires dans le département. Toutes les sociétés politiques recensées en l'an II (57) apparaissent soit directement, soit en épaulant leur municipalité. Seulement une douzaine de faible envergure, dont on ne sait pas grand chose de leur existence, si existence il y a, en l'an II, n'y figurent pas, même pas derrière une municipalité. La seule absence notable est celle de la société populaire de Ganges, ville dont les initiatives patriotiques sont pourtant nombreuses pendant la Révolution. Serait-ce une lacune des sources ou l'indice de faibles liens avec la société populaire de Montpellier, avec des relations essentiellement gardoises ? La géographie a souvent un mot à dire dans les rapports entre sociétés politiques.

Des constatations faites au niveau national se retrouvent ici à l'échelle locale (58) : le don est un des signes de la politisation révolutionnaire qui divise l'espace français entre zones de silence, de refus, de forte implication. Le monde de la plaine, la vallée de l'Hérault, ouverts sur l'extérieur avec de nombreuses sociétés populaires, s'opposent à la bordure montagneuse nord/nord-ouest, plus repliée sur elle-même.

Figure 1 : la répartition des dons pour le vaisseau départemental dans l'Hérault.

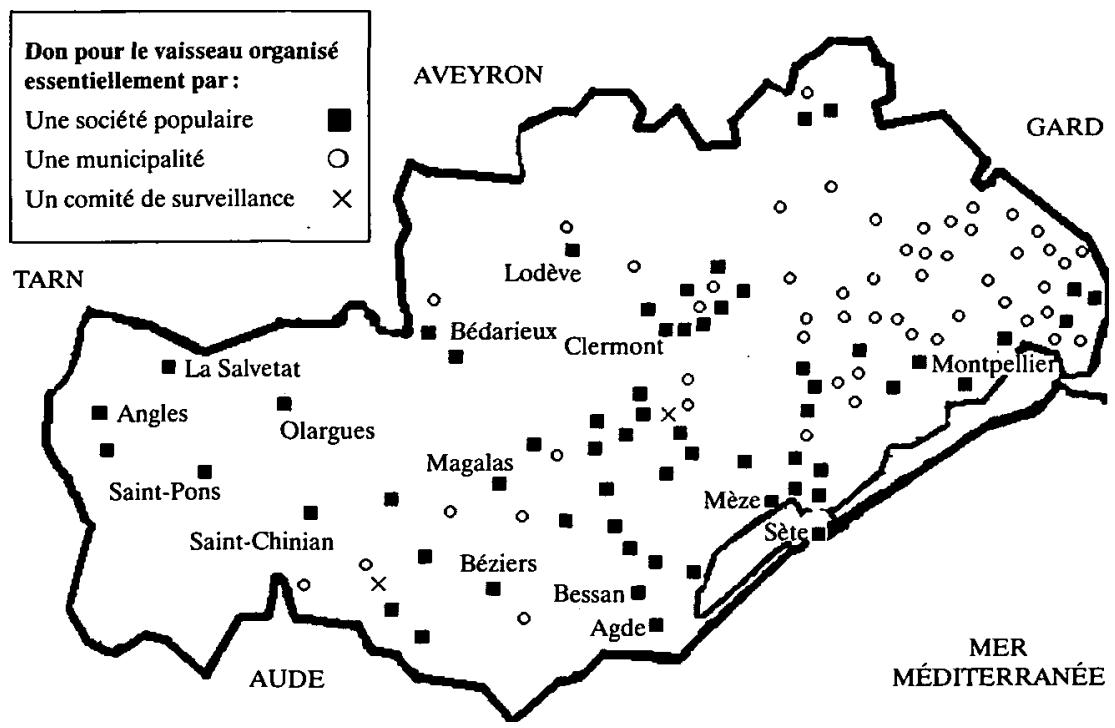

(57) Jean-François DUBOST en a dénombré 91 (« Le réseau des sociétés politiques dans le département de l'Hérault », op . cit. ).

(58) Michel VOYELLE, La découverte de la politique (...), op. cit., p. 256. 
La majorité des offrandes se fait à l'intérieur d'une société populaire, lors d'une séance. La collecte peut se faire le jour même de l'ouverture de la souscription, qui ne dure donc qu'une journée. Plus souvent, elle s'étend sur plusieurs séances, de façon que tous puissent participer, car bien des sociétaires sont peu fortunés ou n'ont jamais d'argent sur eux (59). Le nombre de donateurs est très proche de celui des membres de la société politique. Établir cette relation qui paraît évidente n'est pas toujours chose facile car les listes des sociétaires sont sujettes à variation suivant les époques, et la plupart de celles dont nous disposons aujourd'hui ne datent pas de l'an II mais de l'an III. Enfin, seul un nombre limité de listes complètes de donateurs pour le vaisseau nous sont parvenues [40], provenant d'ailleurs, en majorité, de collectes faites par les municipalités et non pas par les sociétés politiques. Cependant la similitude entre les énumérations des donateurs et celles des membres des sociétés populaires est un fait avéré. À Marseillan, ils sont 167 à donner de l'argent. La liste des membres qui comporte 256 noms en ventôse an II, englobe la quasi-totalité des donateurs (60). Les homonymes rendent souvent difficiles l'identification, mais lorsque le prénom est indiqué dans les deux listes, se retrouvent à chaque fois les mêmes citoyens et comme donateurs, et comme membres de la société populaire (61). Le phénomène est si net que les listes de donateurs, lorsqu'elles ont été conservées, peuvent donner une idée relativement précise des citoyens qui composent la société populaire du cru, même lorsque ses registres et les listes des sociétaires ont disparu. Dire que la liste des donateurs pour le vaisseau départemental de Saint-Jean de Vedas peut donner celle des membres de la société populaire du village, non retrouvée parmi les archives, n'est pas s'aventurer beaucoup, lorsqu'on lit une liste où les premiers noms sont tous accompagnés d'un prénom tiré du calendrier révolutionnaire (62). Dans ces circonstances, la part des donateurs par rapport à la population totale correspond souvent à la proportion de citoyens membres de la société populaire. Deux cas de figure semblent prévaloir.

Lorsqu'il s'agit d'une grande ville, les sociétaires sont une minorité, on le sait (63). Ce qui est frappant, c'est que le don patriotique ne se diffuse

(59) « (...) Nous aurions désiré voir ladite souscription aussitôt remplie qu'ouverte, mais comme la société n'est composée pour la majeure partie que de sans-culottes peu fortunés, nous avons contraints de retarder la fermeture dudit registre jusqu'à ce que tous nos frères ayant pû se procurer de quoi fournir selon leurs facultés. (...) », Ibid., L 5536. Extrait du procès-verbal du 12 germinal an II du conseil général de la commune de Saint-Thibéry « réuni à la société populaire des sans-culottes de la dite commune ».

(60) Ibid., L 4180. Tableau des membres de la société populaire de Marseillan, et ADH, L 3491. Liste des donateurs pour le vaisseau.

(61) Même constatation pour les dons concernant Bessan, Agde, Lunel, et d'autres dons comme ceux concernant la levée de « cavaliers jacobins » à Sète ou à Mèze (ibid., L 3495, L 3491, L 4333, L 4328, L 4180, L 5599).

(62)Ibid., L 3491.

(63) Jean BounER et Philippe BOUTIER, Les sociétés politiques. Atlas de la Révolution française, tome VI, Paris, Éditions de l'EHESS, 1992. 
guère en dehors de la société politique lors de la souscription pour le vaisseau. À Marseillan, Agde, Lunel, Bessan, Lodève, la souscription ne touche vraiment que des sociétaires. Apparemment, les sociétés politiques de villes importantes ne cherchent pas vraiment à élargir le rayonnement du don. Elles affirment qu'elles désirent que toute la population participe, mais cela reste au niveau des déclarations d'intention, affirmation qu'il faut tout de suite nuancer. En effet, si certaines villes comme Lunel affichent des taux très faibles, signe des difficultés des patriotes pour s'affirmer, il n'en est pas de même partout. Lorsque des sociétés populaires comme celles de Bessan ou de Lodève parviennent à faire cotiser $10 \%$ de la population totale, cela signifie que le nombre de citoyens concernés est important, car ces $10 \%$ sont en général des chefs de famille. La notion de foyer est essentielle ici, car nombre de donateurs insistent sur l'aspect familial de leur apport. La liste de La Salvetat donne des noms d'individus mais aussi, à côté du chef de famille, le reste de sa maisonnée : «Reinaud et sa famille», «Alexis Gros et sa famille »,' « Gozet père fils et neveu », « Negre sa famille », « Montai sa mère et sa tante », «Goutines neveu et ses sœurs » etc. (64). Plus qu'un individu qui offre, c'est une structure familiale qui montre son patriotisme.

Le phénomène est encore plus net dans certaines bourgades. Là où la «ville » est plus modeste, la part des donateurs et des membres de la société populaire augmente, au moins pour les bourgs les plus politisés. Certains gros villages sont caractérisés en effet par une société populaire qui englobe pratiquement tous les chefs de famille. À Paulhan, sur une population de 1188 habitants environ, 198 participent à la souscription, ce qui correspond, en gros, au nombre de membres de la société politique du lieu (65). Le don touche un peu moins de $20 \%$ de la population soit les chefs de famille du village ici : rares sont les foyers qui ne participent pas au don. La même constatation peut être faite pour une bourgade comme celle de Poussan, où plus de $16 \%$ de la population fait une offrande. Les contemporains tout en affirmant la liberté du citoyen, ne le conçoivent qu'intégré dans une cellule familiale et dans une communauté. Pour les municipalités comme les sociétés populaires, la notion de « feux » reste largement utilisée comme mode de calcul de la population (66). En prenant en compte cet élément, il apparait que la pratique du don patriotique touche plus de personnes que les listes de donateurs peuvent le laisser croire, puisque derrière le donateur se trouve souvent une famille entière.

(64) ADH, L 5536. État des dons de la société populaire de la commune de la Salvetat.

(65) Ibid., L 5599. Liste des membres qui composent la société populaire de Paulhan ; ibid., L 3455 Liste des donateurs pour le vaisseau.

(66) Cf. par exemple la lettre de la société populaire de Courmonsec à celle de Montpellier à propos du don pour le vaisseau : « (...) Quoique la commune ne contienne que 60 feux et que la société de 20 à 25 républicains, cette levée a produit plus de 600 livres » (ibid., L 5532). 
Par voie de conséquence, la participation des femmes au don patriotique est moins importante qu'on ne le dit généralement, au moins pour les offrandes pécuniaires. Si elles participent, c'est dans l'ombre de leur époux. Pour des offrandes comme celles de charpie destinées aux hôpitaux, ce sont en majorité des femmes et des enfants qui se manifestent dans les séances des sociétés populaires. En revanche, leur part est singulièrement réduite dès qu'il s'agit d'argent. Les listes conservées, qu'elles proviennent des municipalités ou des sociétés politiques, prouvent cette assertion en maints endroits. À Lavèrune, 89 personnes, dont 14 femmes, participent à la souscription pour le vaisseau (67). Or sur ces 14 noms de femmes, dans la liste, 12 sont précédés du mot « veuve ». Cela signifie que les participants agissent en tant que chef d'une maisonnée, celui qui détient l'argent. À Paulhan, qui se distingue par une forte participation de la population, aucune femme n'apparaît parmi les donateurs (68). Bien sûr, ce n'est pas le cas partout. Des listes font apparaitre l'apport des femmes et des enfants. Des membres des sociétés populaires insistent sur l'action des citoyennes, au point que la société politique tient à s'effacer devant la générosité des femmes patriotes qu'elle met en valeur (69). Notre échantillon n'est pas assez important pour apporter des réponses définitives, mais d'après les listes dont nous disposons, il semblerait que la présence des femmes soit la marque des sociétés populaires les plus politisées. Ce n'est peut-être pas un hasard si, en proportion, la liste qui comporte le plus de prénoms révolutionnaires soit une de celles où l'on trouve le plus de femmes qui, apparemment, ne sont pas des veuves (70).

Mais les sociétés populaires ne sont pas seules à organiser la levée. De nombreuses municipalités interviennent aussi. Les communes qui participent, sans avoir de société politique, sont pour la majorité situées dans le district de Montpellier. L'aire d'influence du chef-lieu de département et de sa société populaire se fait clairement sentir, d'où un net déséquilibre de la répartition de l'offrande sur le territoire du département : plus on s'éloigne de Montpellier, moins l'on trouve de municipalités qui participent au don. Il

(67) Ibid, L 3495. «État des citoyens de lavèrune qui ont donné pour la construction du vaisseau de la République portant le nom du département de l'Hérault. »

(68) Ibid., L 5599. On pourrait multiplier les exemples d'après les listes des donateurs (les prénoms étant généralement indiqués, le risque d'erreurs est minime) : à Saint-Christol, 47 donateurs, dont deux femmes qui sont veuves (ibid., L3491); à Marseillan, 170 offrandes sont faites, dont 5 par des femmes (ibid, L 4328); à Grabels, parmi les 58 donateurs, deux femmes, des veuves (ibid., L 3495).

(69) Ibid., L 5503. Séance de la société populaire de Montpellier du 16 floréal an II accueillant une députation de la société populaire de Clermont l'Hérault dont l'un des commissaires fait un discours : «L'orateur (...) observe de plus à la société que les citoyennes ont beaucoup contribué à cette heureuse collecte (...) l'assemblée a délibéré mention honorable l'accolade fraternelle à la commission et les honneurs de la séance et la mention honorable aux citoyennes de Clermont. » Dans un tel cas, on peut penser qu'il y a des femmes parmi les commissaires de Clermont reçus à Montpellier.

(70) Celle de Saint-Jean de Vedas : 78 donateurs, 13 noms ou prénoms révolutionnaires au moins ( 1 «La Raison », 3 « Romarin », 2 « Sainfoin », 2 « Primevère », 4 «Potiron », 1 «Concombre ») et 10 femmes (ibid.,L 3491). 
est à noter que dans deux cas au moins, à Belarga et à Puisserguier, le comité de surveillance du lieu organise la levée du don, en collaborant plus ou moins avec la municipalité, voire la société populaire. Par l'organisateur du don, il est possible de discerner quelle est l'institution qui dirige le bourg à cette époque. En règle générale, là où elle existe, c'est la société populaire qui prend les rênes. Mais il apparaît en maintes occasions, comme à SaintThibéry déjà évoqué, que société politique et municipalité se trouvent sur un pied d'égalité, quand ce n'est pas la seconde, qui, quelquefois, assume l'essentiel de la mobilisation patriotique. Parmi les municipalités, deux types de comportements sont discernables.

Dans nombre de petites communes, le nombre de donateurs est faible. L'absence d'une société populaire a des répercussions sur le don. Il est vrai aussi que les communes qui n'ont pas de sociétés politiques sont les plus modestes en population et en ressources. Dans ces conditions, le don se limite souvent aux officiers municipaux. À Combaillaux, dans la liste des dons, ce sont eux qui apparaissent, derrière l'agent national et le maire qui sont les plus gros donateurs avec une offrande respective de 10 et de 5 livres (71). À Boisseron, à Sussargues, le même phénomène est sensible : le maire et l'agent national sont les premiers de la liste, suivis par le reste du conseil général de la commune (72). Le nombre de donateurs en ces communes est limité : une dizaine de noms le plus souvent. Le don en ce cas est nettement l'affaire de municipalités qui, pour afficher leur patriotisme, se sentent obligées de participer à la souscription. Elles ne tentent aucunement de faire appel au reste de la communauté. La levée est faite immédiatement après lecture de l'adresse expédiée par la société populaire de Montpellier ou l'agent national de leur district. Il est à noter cependant que des villages, pourtant proches de Montpellier, n'ont pas cru participer au don pour le vaisseau.

L'absence d'une société populaire dans une commune ne signifie pas obligatoirement la faiblesse de l'engagement patriotique. Dans certaines communautés, plutôt modestes, le cercle des donateurs ne se limite pas à la municipalité. À Montarnaud, village de 400 habitants environ, situé dans le canton de Saint-Georges d'Orques, le nombre de donateurs dépasse la cinquantaine. À Candillargues, village de 114 habitants environ, 31 personnes participent (73). Le fait que dans la plupart des cas ce sont des hommes, des chefs de famille, implique un nombre important de personnes, même si c'est indirectement, selon le même phénomène observé pour les sociétés populaires.

(71) Ibid., L 3491. «Levée pour un vaisseau de ligne. Liste des citoyens qui donnèrent. »

(72) Ibid., liste des donateurs des dits villages.

(73) Ibid, et L 3495. Liste des donateurs de Montarnaud. Les chiffres de population utilisés ici son tous tirés de Claude MOTTE, Paroisses et communes de France. Dictionnaire d'histoire administrative et démographique, 34, Hérault. Paris, Éditions du CNRS, 1989. (Ce sont les chiffres de l'an HI qui ont été choisis plutôt que ceux de 1792.) 
L'implication patriotique par le don, avec ces premières observations, est un phénomène variable selon les communes concernées : soit une affaire de notables et /ou d'une poignée de patriotes, soit un geste qui rassemble une partie non négligeable des citoyens (et citoyennes) d'une communauté. Cette diversité sous-entend la liberté du don révélateur de la mobilisation patriotique des régions ou des citoyens évoqués.

La même variété est présente dans les différentes sommes versées selon les individus. Viennent d'abord ce qui peut être qualifié d'offrande symbolique, les sommes inférieures à une livre, et à la limite, celles qui n'atteignent pas les cinq livres. Dans les sociétés populaires où les dons sont nombreux, comme à Bessan, ces sommes constituent une large majorité.

\section{Sommes versées par donateur}

Figure 2 : à Lunel

Nombre de donateurs

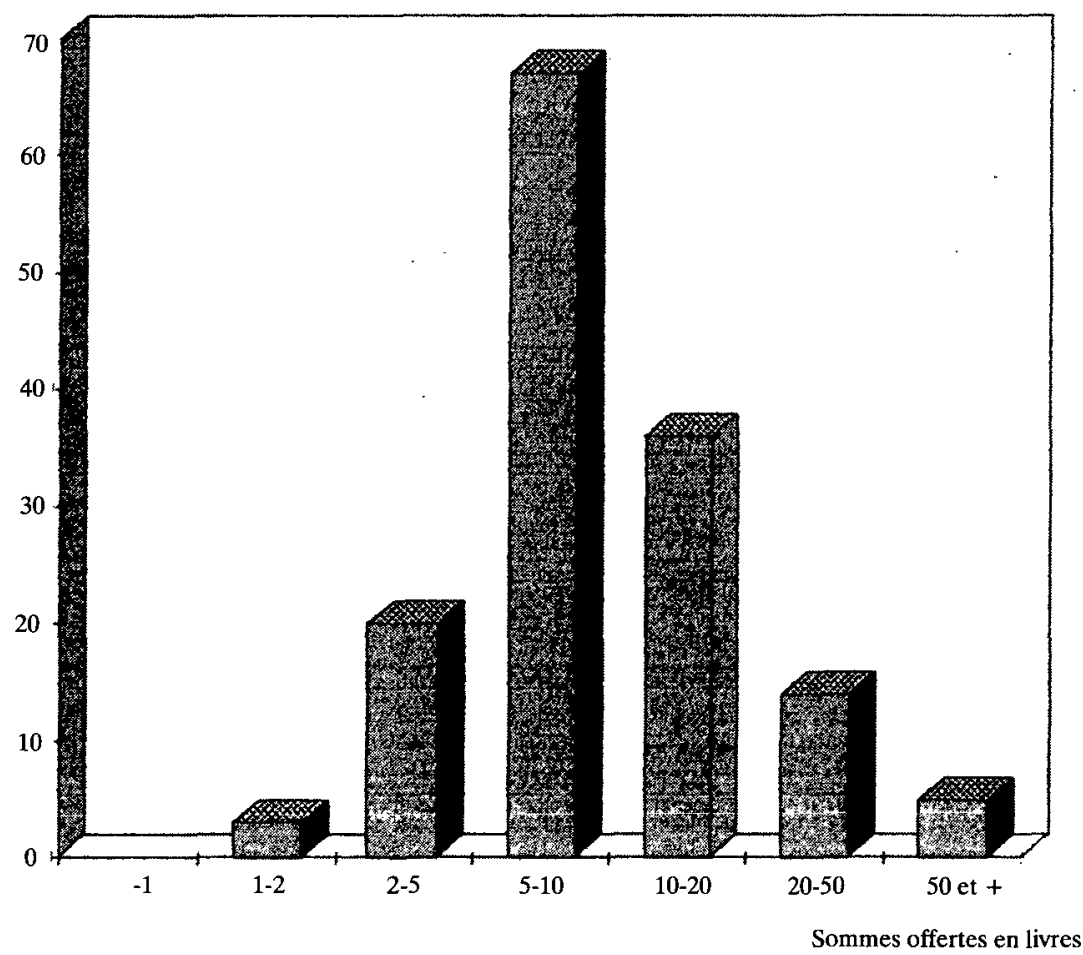


Figure 3 : à Bessan

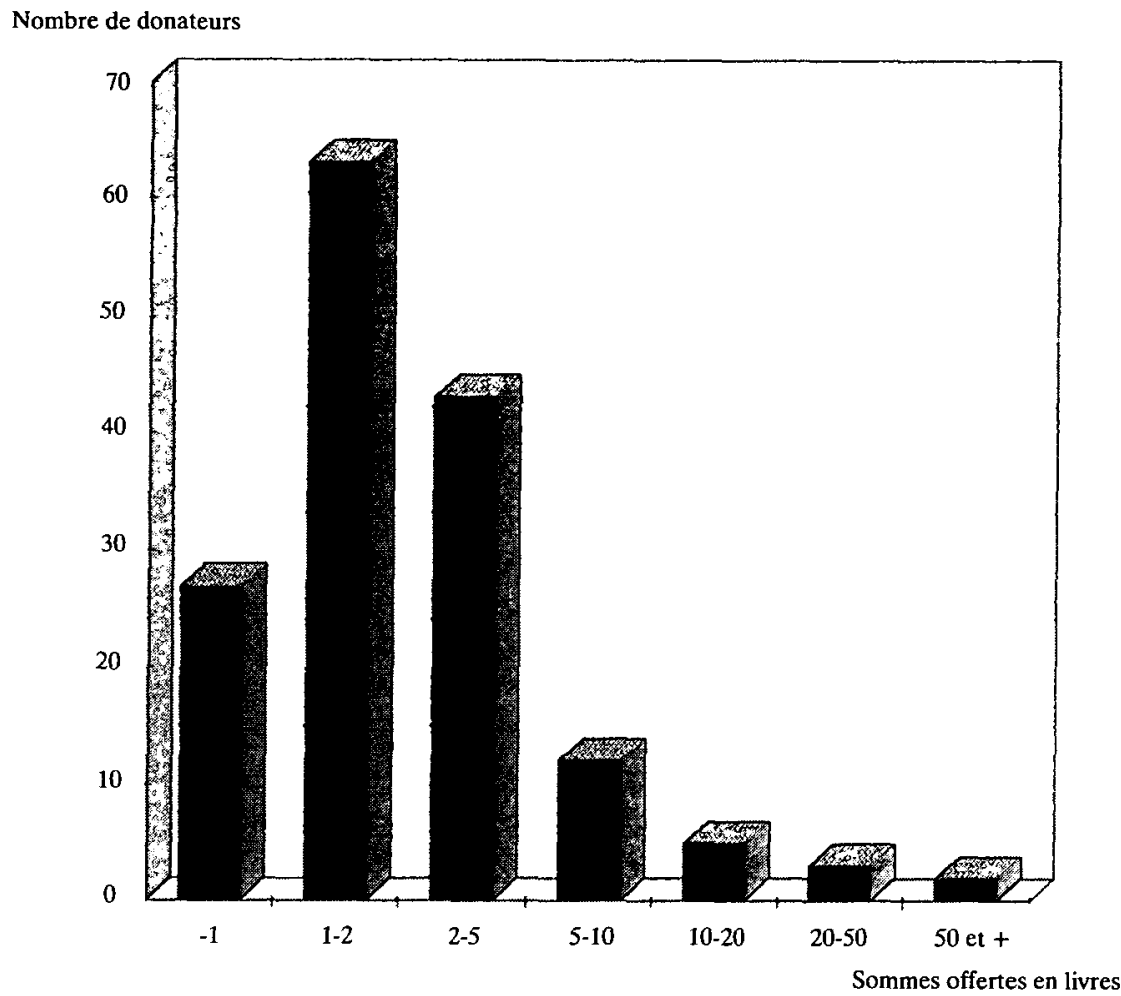

Mais dans d'autres communes ou sociétés populaires, les dons se caractérisent par des offrandes individuelles plutôt conséquentes qui quittent le terrain symbolique. À Agde, où le nombre de donateurs est important (407), proportionnel à une population nombreuse pour le littoral (presque 6500 habitants), les dons supérieurs à 5 livres sont majoritaires. La même constatation s'impose à Lunel, voire à Marseillan (74).

Plus la société populaire ou la ville est grande, plus les offrandes élevées sont nombreuses, correspondant au niveau de fortune de l'ensemble urbain. À Lodève cela se vérifie, avec des citoyens, surtout les fabricants, qui n'hésitent pas à verser de fortes sommes. 
Figure 4 : à Lodève

Nombre de donateurs

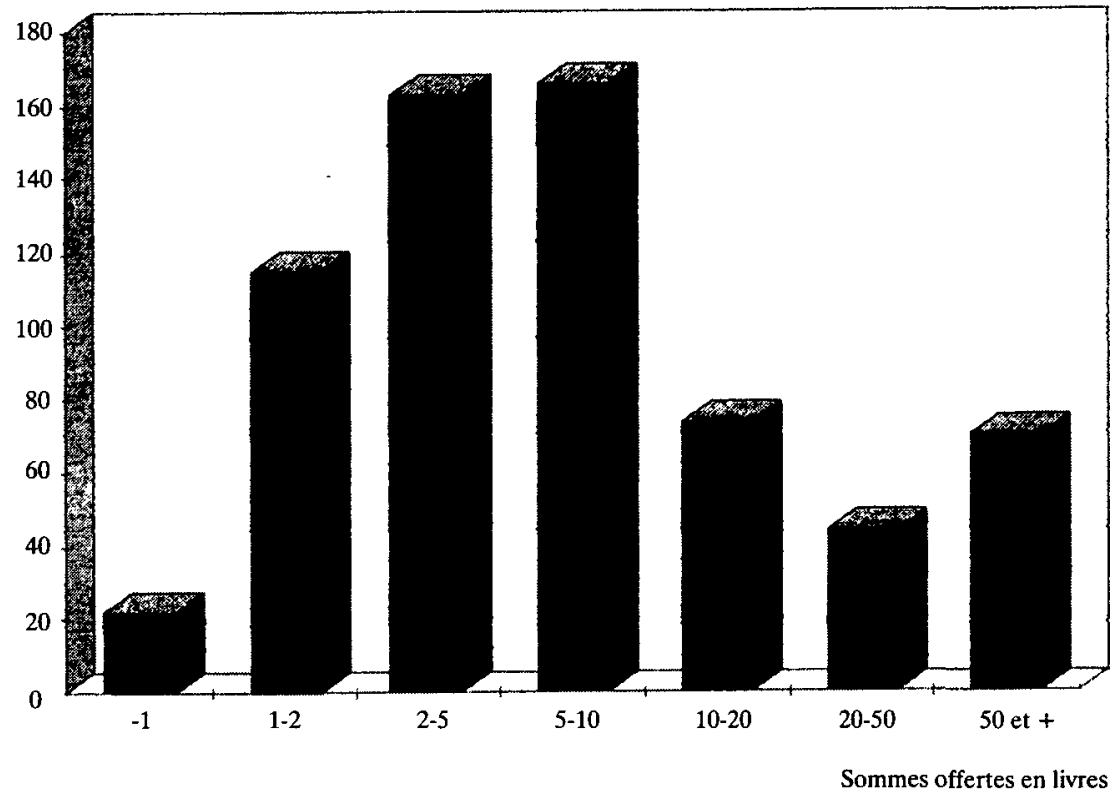

Les manufacturiers les plus en vue dépassent les 200 livres, avec certains qui n'hésitent pas à aller bien au-delà, tel un François Salze qui offre 2000 livres (75). Dans ces conditions, les administrateurs, en tête dans les autres communautés, ne peuvent être qu'en retrait ici. Plusieurs membres du district offrent 50 livres, ce qui les placerait largement parmi les donateurs les plus généreux en maintes bourgades. Cela n'est pas le cas à Lodève, riche en fabricants aisés, pour qui la Révolution, avec la reprise des commandes militaires, est chose bénéfique, au moins dans un premier temps. Seul un administrateur qui est aussi fabricant, comme M. Fabreguettes, issu d'une des manufactures les plus importantes de la ville, peut figurer parmi les gros donateurs, avec 300 livres (76). Lodève se caractérise dans le département par une proportion conséquente de ces donateurs importants, ce qui ne se retrouve guère qu'à Montpellier, autre ville riche et puissante du département (77). L'analyse des dons patriotiques permet donc de discerner les zones les plus

(75) Ibid., 93 EDT 2 113. « Souscription volontaire pour fournir à la construction d'un vaisseau de ligne...»

(76) Ibid.

(77) Ibid,L 5502-5504. 
patriotes mais donne aussi l'indice de prospérité d'une communauté. La répartition des sommes versées reflète aussi la structure la société populaire : le niveau de fortune entre celle d'un bourg rural composé d'agriculteurs n'a rien de comparable avec celle d'une ville riche en manufactures. Mais la taille et la richesse n'expliquent pas automatiquement l'ampleur d'un don. La comparaison entre le nombre d'habitants et le don offert par la communauté souligne de nettes différences d'attitudes qui reflètent le degré de l'engagement patriotique. Si les villes les plus importantes se distinguent par les plus fortes sommes, la diversité extrême de la pratique du don apparaît une fois encore. Ainsi Lunel, ville prospère de l'est du département, 4170 habitants, offre 1808 livres, ce qui la met au même niveau que la bourgade voisine de Mauguio (1821 livres pour 1719 habitants seulement), sans parler de Marsillargues, peuplée de 3000 habitants environ, qui donne 6000 livres. La même diversité est la règle dans les villages. Quoi de plus dissemblable que Courmonsec, 439 habitants, dont l'offrande s'élève à 600 livres, tandis que Bouzigues n'en offre que 382 pour une population deux fois plus nombreuse ? L'exemple le plus extrême est celui de La Salvetat, composée de plus de 3000 habitants, qui dépasse de peu les 200 livres, chiffre atteint par beaucoup de modestes bourgades (78). La richesse compte peut-être moins que l'engagement patriotique, ce qui expliquerait ces différences entre les localités. Concrètement, cela signifie que c'est la liberté du don qui prévaut. Si cela est vrai pour les différents villages ou villes, cela doit se vérifier en comparant les offrandes des différents citoyens. Il est difficile de confronter le montant du don avec le niveau social du donateur. Les rôles de contribution datent souvent de 1792, ce qui complique la tâche avec la présence d'homonymes, la pratique des surnoms. Ils sont pourtant les points de comparaison les plus intéressants, dans un monde où l'essentiel de la richesse vient de la terre. C'est le cas à Paulhan, où la majorité des membres de la société populaire, qui se confondent avec les donateurs pour le vaisseau, sont «agriculteurs » (79). Un échantillon d'une cinquantaine de donateurs est pris en compte ici, soit un peu moins du quart. Le choix de ces individus a été dicté par le souci d'écarter tout risque de confusion avec un homonyme ou une mention peu explicite. L'hétérogénéité prévaut : des paysans désignés comme «manouvriers » ou «travailleurs », dont le rôle de contribution attribue de faibles revenus et un impôt dérisoire, font des dons qui sont équivalents à ceux offerts par des « ménagers », paysans propriétaires plutôt aisés. Cela est vrai à Paulhan, mais cela se vérifie aussi dans d'autres bourgs, comme à Marseillan (80).

(78) Ibid,L 5502-5504.

(79) Ibid., L 5599. Liste des donateurs de Paulhan pour le vaisseau et liste des membres de la société populaire de Paulhan (cette dernière est utilisée car elle permet de recouper les informations concernant la profession des donateurs). Ibid., L 4845. Matrice des rôles de la contribution foncière de Paulhan (1792).

(80) Ibid., L 4342. Contribution foncière de Marseillan (1792) et ibid., L 4328. Liste des dons versés pour le vaisseau. 
De modestes «brassiers » font des dons de faible envergure qui n'en constituent pas moins un sacrifice vu leurs ressources : comme les autres citoyens, ils entendent concourir à la défense de la patrie. Cela est l'indice d'un engagement patriotique. Quoi qu'en disent les textes des sociétés populaires qui affirment qu'il faut faire contribuer les « riches », les propriétaires les plus aisés ne sont apparemment pas obligés de se sacrifier. Ils donnent certes, mais comme les autres membres de la communauté, le plus souvent un peu plus que la moyenne, mais pas toujours. L'offrande est loin de remettre en péril un équilibre financier. La pression ne vient d'ailleurs pas le plus souvent des «pauvres» de la commune qui les obligeraient à contribuer. La lecture des procès-verbaux des séances de la société populaire de Lodève montre de façon très nette que les fabricants, c'est-à-dire les citoyens les plus aisés de la commune, font des dons importants pour en remontrer à leurs concurrents. Une véritable émulation, que l'on peut qualifier de surenchère, se développe (81). La volonté de se montrer le plus généreux pour sa patrie est clairement dictée par un souci de prestige social. $\mathrm{Ne}$ pas donner beaucoup, est ressenti comme déchoir un peu de sa position. La

Figure 5: Comparaison entre l'offrande et le niveau social du donateur à Paulhan (dons inférieurs à 20 livres)

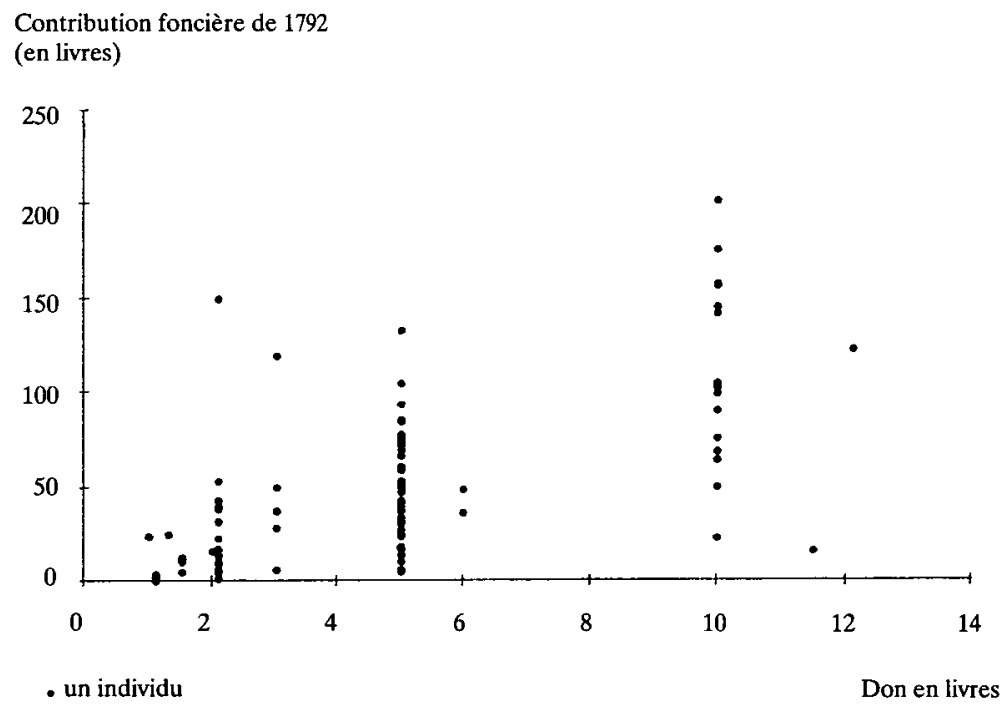

(81) Voir particulièrement la séance de la société populaire de Lodève du 13 germinal an II (ibid., L 8812. Procès-verbaux des séances de la société.) 
pression de la communauté vient davantage des hiérarchies traditionnelles que d'une idéologie propre à l'an II.

À croire que le succès du don patriotique en l'an II s'explique parce qu'il a su se fondre dans des pratiques anciennes. Les sociétés populaires n'inventent rien. Les souscriptions qu'elles réalisent sont très proches de celles qui sont organisées dans les villages, sous l'Ancien Régime, comme au $\mathrm{XIX}^{\mathrm{e}}$ siècle, pour une œuvre qui intéresse la communauté tout entière, telle la fonte d'une cloche. "L'histoire campanaire », étudiée par Alain Corbin, recèle une multitude de souscriptions qui rappellent fortement les initiatives des sociétés populaires (82). La souscription soude là comme ici une communauté. Les villageois insistent sur les droits que confère l'offrande à chaque donateur, quel que soit le niveau du don. Pauvre ou riche, chacun devient moralement propriétaire de la cloche du village, comme chaque citoyen de l'an II obtient sa part de souveraineté par l'exercice du don. Le gros donateur, noble ou propriétaire aisé, n'a pas plus de droits sur la cloche que le pauvre qui a modestement participé. Il en reçoit seulement une marque d'honneur supplémentaire, tel le nom sur la cloche. Cela rappelle les «mentions honorables» des sociétés populaires, façon de valoriser également le don important. L'affichage des listes de donateurs par les sociétés populaires n'offre pas non plus de singularité. La liste des souscripteurs pour la cloche du village est affichée bien en vue, sur la porte de l'église, tandis que les récalcitrants subissent des pressions pour s'exécuter (83). L'opprobre est jeté sur ceux qui refusent de mettre la main à la poche (84). Le refus passe pour un rejet de la communauté, de laquelle on s'exclut finalement de soi-même, en ne communiant pas autour de valeurs ou d'un but commun. Cet affichage fait inévitablement penser aux pratiques des sociétés populaires, qui mettent en valeur les citoyens donateurs, sur une liste d'honneur, bien en vue, avec parfois, en face ou à côté, " une liste d'infamie » où sont notés ceux qui ont refusé de donner (85). Sur ces listes, tous les dons sont scrupuleusement recensés, les modestes sols du brassier comme les centaines de livres du propriétaire. Comment enfin ne pas mettre en parallèle les dons d'objets métalliques, en cuivre, fers, argents, sous forme de vaisselle, vieux chenets, casseroles..., offerts spontanément par les villageois pour la cloche paroissiale et les dons de l'an II pour permettre de

(82) Alain CORBIN, Les cloches de la terre. Langage sonore et culture sensible dans les campagnes au XIX siècle, Paris, Flammarion, 1994, notamment la partie II, «L'esprit de clocher, 1) la sonnerie communautaire, un marqueur symbole d'identité». Alain Corbin centre son étude sur le XIX siècle, mais il mentionne aussi des souscriptions réalisées très tôt au XVIII' siècle, comme à Basse-Vigneulles (Moselle), en 1710 .

(83) Id. Une liste est apposée sur la porte de l'église de Saint-Louis-les-Phalsbourg par exemple.

(84) Id. Les nouvelles cloches ne sonneront pas, lors des cérémonies, pour ceux qui ont refusé de participer à la souscription, y compris pour les enterrements.

(85) Ainsi, dans la société populaire de Lodève, une fois la souscription pour le vaisseau clôturée, « une liste des sociétaires qui n'ont rien donné » devra être «formée et affichée » dans la salle des séances. (ADH, L 8812. Séance du 3 prairial an II.) 
forger de nouvelles armes ou construire un vaisseau ? Il est bien tentant de faire la même mise en relation pour l'offrande de bijoux pour financer les combats de la Révolution et le jet, dans le moule de refonte de la cloche, au moment de la fusion, de pièces d'or ou d'anneaux censés donner un meilleur son (86). Que cette dernière pratique soit réelle, ou légendaire, peu importe. Le fait reste que dans l'esprit des villageois, le don ne peut se limiter à une offrande pécuniaire. Le donateur doit payer de sa personne, sacrifier un objet qui signifie sa fusion avec l'ensemble de ses concitoyens. La notion de sacrifice est inséparable du don.

\section{Conclusion}

La diffusion du don patriotique est un trait caractéristique de l'an II. Le don permet de discerner la pratique du patriotisme et de la citoyenneté en Révolution. La diversité de son empreinte sur le terrain indique l'aspect volontaire de ce geste en général, en mettant en valeur les zones patriotes. Son ampleur, même si les offrandes n'ont pas toujours abouti à des réalisations concrètes, est une des clefs qui expliquent la réussite de la mobilisation idéologique de l'an II. Le don indique à la fois le succès des sociétés populaires à diffuser les principes patriotiques (égalité, fraternité, défense du pays) mais aussi les limites de leur action, car elles ne parviennent pas toujours à franchir, notamment en ville, le cercle plutôt restreint de leurs sociétaires. Les patriotes réussissent le mieux là où ils parviennent à marier engagement révolutionnaire et pratiques anciennes. Le don est l'indice d'une solidarité et d'une identité commune qu'il renforce : les sociétés populaires de l'Hérault, qu'elles le veuillent ou non, s'inscrivant par leur offrande pour un vaisseau «sans-culotte» dans la lignée des États de Languedoc. Le don commun s'enracine profondément dans l'existence des communautés villageoises. La société populaire assume, dans sa pratique de l'offrande patriotique, le rôle de la communauté d'habitants : en l'an II, le succès des dons ne peut s'expliquer que par cet aspect, avec des sociétés politiques qui parviennent à transcender la fierté et les solidarités locales en appartenance nationale.

Nathalie ALZAS agrégée d'histoire chargée de cours à l'Université de Provence

Champigonnet Salavas 07150 Vallon-Pont d'Arc

(86) Alain CORBIN, Les cloches de la terre...,op. cit. 
Figure $6:$ Nombre de donateurs par rapport à la population de la localité ( $M=$ municipalité; $S P=$ société populaire $)$

\begin{tabular}{|c|c|c|c|c|}
\hline Localité & $\begin{array}{c}\text { Nombre } \\
\text { d'habitants }\end{array}$ & $\begin{array}{l}\text { Nombre de } \\
\text { donateurs }\end{array}$ & $\begin{array}{c}\text { Part des donateurs } \\
\text { dans la population } \\
\text { totale }\end{array}$ & $\begin{array}{l}\text { Structure qui a } \\
\text { organisé la levée }\end{array}$ \\
\hline LA SALVETAT & 3813 & 34 & $0,8 \%$ & SP \\
\hline FABREGUES & 624 & 11 & $1,7 \%$ & $\mathbf{M}$ \\
\hline PUIMISSON & 380 & 11 & $2,8 \%$ & $\mathbf{M}$ \\
\hline MAGALAS & 904 & 27 & $2,9 \%$ & $\mathrm{SP}(69)^{*}$ \\
\hline LUNEL & 4170 & 160 & $3,8 \%$ & SP $(192)^{*}$ \\
\hline BOISSERON & 195 & 10 & $5,1 \%$ & $\mathbf{M}$ \\
\hline MARSEILLAN & 3210 & 170 & $5,2 \%$ & $\mathrm{SP}(256)^{*}$ \\
\hline SAINT-SERIES & 145 & 6 & $5,2 \%$ & $\mathbf{M}$ \\
\hline AGDE & 6744 & 407 & $6,0 \%$ & $\mathrm{SP}(453)^{*}$ \\
\hline ROUJAN & 1011 & 87 & $8,6 \%$ & $\mathbf{M}$ \\
\hline SAINT-JEAN-DE-VEDAS & 799 & 78 & $9,7 \%$ & SP \\
\hline LODEVE & 7906 & 813 & $10,2 \%$ & SP \\
\hline MONTFERRIER & 273 & 28 & $10,2 \%$ & $\mathbf{M}$ \\
\hline BESSAN & 2003 & 213 & $10,6 \%$ & $\mathrm{SP}(172)^{*}$ \\
\hline CAZEVIELLE & 74 & 8 & $10,8 \%$ & $\mathbf{M}$ \\
\hline VENDARGUES & 426 & 48 & $11,2 \%$ & $\mathbf{M}$ \\
\hline COMBAILLAUX & 157 & 18 & $11,4 \%$ & $\mathbf{M}$ \\
\hline BEAUZULE-DE-MONIMEL & 340 & 40 & $11,7 \%$ & $\mathbf{M}$ \\
\hline MONTARNAUD & 417 & 51 & $12,2 \%$ & $\mathbf{M}$ \\
\hline SAINT-CHRISTOL & 375 & 47 & $12,5 \%$ & $\mathbf{M}$ \\
\hline TEYRAN & 190 & 26 & $13,6 \%$ & M ou SP \\
\hline SATURARGUES & 202 & 28 & $13,8 \%$ & $\mathbf{M}$ \\
\hline COURMONSEC & 340 & 50 & $14,7 \%$ & $\mathbf{M}$ \\
\hline GRABELS & 381 & 58 & $15,2 \%$ & $\mathbf{M}$ \\
\hline SAINT-GELLY & 305 & 48 & $15,7 \%$ & $\mathbf{M}$ \\
\hline LUNEL-VIEIL & 561 & 95 & $16,2 \%$ & $\mathbf{M}$ \\
\hline POUSSAN & 1319 & 220 & $16,6 \%$ & SP \\
\hline VERARGUES & 84 & 14 & $16,6 \%$ & $\mathbf{M}$ \\
\hline LES MATELLES & 296 & 50 & $16,8 \%$ & $\mathbf{M}$ \\
\hline CLAPIERS & 168 & 30 & $17,8 \%$ & $\mathbf{M}$ \\
\hline GUZARGUES & 73 & 13 & $17,8 \%$ & $\mathbf{M}$ \\
\hline LAVERUNE & 493 & 89 & $18,0 \%$ & $\mathbf{M}$ \\
\hline ASSAS & 197 & 36 & $18,2 \%$ & $\mathbf{M}$ \\
\hline VAILHAUQUES & 228 & 43 & $18,8 \%$ & $\mathbf{M}$ \\
\hline RESTINCLIERES & 146 & 28 & $19,1 \%$ & $\mathbf{M}$ \\
\hline PAULHAN & 1019 & 198 & $19,4 \%$ & SP $(209)^{*}$ \\
\hline $\begin{array}{l}\text { BAILLARGUES } \\
\text { et COLOMBIERS }\end{array}$ & 455 & 89 & $19,5 \%$ & $\mathbf{M}$ \\
\hline MONTOULIERS & 230 & 51 & $22,1 \%$ & M \\
\hline AGONES & 92 & 21 & $22,8 \%$ & $\mathbf{M}$ \\
\hline CANDILLARGUES & 114 & 31 & $27,1 \%$ & $\mathbf{M}$ \\
\hline
\end{tabular}

* (entre parenthèses, nombre de membres de la société populaire) 